\title{
Déficit de Integración Sensorial: Efectos a largo plazo sobre la ocupación y el juego
}

\author{
I mperatore Blanche, Erna \\ Assistant Profesor of Clinical Practice, Department of Occupational Science and \\ Therapy, University of Southern California. \\ Contacto > > blanche@usc.ed, impe@aol.com Fono (310)892-3176 \\ Referencia > > Imperatore Blanche,Erna. "Déficit de Integración Sensorial: \\ Efectos a largo plazo sobre la ocupación y el juego". Revista Chilena de Terapia \\ Ocupacional. №5. Noviembre 2005.
}

\section{- Resumen}

El impacto de los déficit de integración sensorial sobre las ocupaciones ha sido ampliamente descrito en niños. Sin embargo, su efecto a largo plazo, en adolescentes y adultos, ha permanecido más velado.

Este documento enfoca las consecuencias de este problema en el futuro del individuo, que deben ser tomadas en cuenta por la Terapia Ocupacional al tratar a niños con déficit de integración sensorial.

Respecto a la influencia del déficit de integración sensorial sobre las ocupaciones futuras, podemos apreciar dos tipos de patrones. El primero, de búsqueda de sensaciones y actividades de alto riesgo, aparece en personas que durante su infancia han presentado hiposensitividad en el sistema vestibular y propioceptivo, y dificultades de modulación. El segundo, de desorganización, se observa en individuos que presentaron dificultades en programación motora o dispraxias. Estos patrones deben ser identificados temprano en la vida, para que la intervención tenga un impacto en la narrativa futura del niño.

\section{- Abstract}

The impact of sensory integration dysfunction on the occupational performance of children has often been described, however the long term impact of these dysfunctions on the life of adolescents and adults is seldom mentioned. The present article focuses on the possible long term consequences of sensory integration dysfunctions derived from the literature. These long term consequences need to be considered when providing occupational therapy for children.

In reference to the long term effects of SI dysfunctions two patterns emerge from the literature: sensation seeking, often observed in individuals who exhibited signs of hyporesponsiveness to vestibular and proprioceptive input and sensory modulation disorders as children; and the second, clumsiness and disorganization that is observed in individuals who exhibited signs of dyspraxia as children. These patterns need to be identified early in life so intervention can affect the child's future narrative. 


\section{I ntroducción}

La Ciencia de la Ocupación ha sido descrita como el estudio de la forma, función y significado de la Ocupación. La forma se refiere a lo observable de la Ocupación y toma en cuenta lo que una persona o un grupo de individuos ejecutan y las circunstancias bajo las cuales lo hacen. La función se refiere al nexo entre ocupación y salud, estado de bienestar, funcionamiento diario, desarrollo de la maestría y calidad de vida. Por último, el significado alude a la experiencia que aportan las distintas ocupaciones, a las bases de elección de las mismas y a la importancia o valor que tienen para cada persona ${ }^{(1)}$.

Yerxa ${ }^{(2)}$ asegura que la Terapia Ocupacional es importante en el proceso de entrega de un repertorio de actividades que la persona puede desarrollar durante su vida. Esta selección le ayuda al individuo a permanecer saludable y resistir el stress. Cuando tratamos niños con déficit de integración sensorial es importante ayudar al paciente a seleccionar ocupaciones y a crear un repertorio de ocupaciones que proveen equilibrio y promueven la salud a través de las experiencias sensoriales. Por lo tanto, en la intervención con niños no sólo debemos enfocarnos en la forma de las respuestas adaptativas a corto plazo y dentro de nuestro ámbito de trabajo, sino también en la funcion y significado de las respuestas adaptativas a a largo plazo y las ocupaciones en las cuales el paciente participará individualmente en su comunidad ${ }^{(3)}$.

\section{Déficit a largo plazo "}

Los efectos a largo plazo del déficit de integración sensorial han sido estudiados en niños con déficit de integración sensorial y en pequeños con déficit en coordinación motora y déficit atencional. Se recurre a la literatura que describe el desarrollo de niños con déficit atencional y déficit en coordinación motora, pues niños que presentan este diagnóstico suelen manifestar déficit de integración sensorial ${ }_{-}^{(4)}{ }^{(6)}$. Además, existe literatura dentro de Psicología que describe rasgos de personalidad que incluyen la búsqueda de sensaciones. Esta literatura también aporta al entendimiento de los problemas a largo plazo de niños con déficit de integración sensorial.

Una importante investigación del efecto del déficit en integración sensorial sobre los logros académicos a largo plazo fue hecho por Parham en $1998^{(7)}$. En este estudio longitudinal, Parham hizo un seguimiento durante cuatro años a 32 niños que presentaban déficit de aprendizaje y a 35 que no lo tenían, evaluándolos en sus funciones de integración sensorial, en inteligencia y en nivel académico. Al inicio de este estudio los niños tenían entre 6 y 8 años. Tras cuatro años, volvió a evaluarlos y vio que se puede recurrir a los factores de integración sensorial para predecir el funcionamiento académico en matemáticas y lectura. En especial, las funciones de praxis tuvieron un valor predictivo alto con relación a matemáticas. Es importante destacar que los niños con déficit de aprendizaje no superaron sus problemas en percepción visual y praxis y por lo tanto estos problemas deben ser tratados lo más tempranamente posible ${ }^{(7)}$.

Otro estudio longitudinal de Rasmussen y Gillberg ${ }^{(8)}$, apoya la idea de que los problemas en los niños no desaparecen con la edad. En este estudio, los investigadores hicieron un seguimiento clínico de niños con déficit atencional y déficit de coordinación motora por aproximadamente 18 años. Cuando cumplieron 22 años, los resultados comprobaron que estos niños no superan los problemas con la edad y pareciera ser que estos diagnósticos son los datos predictivos más importantes para explicar el pobre funcionamiento sicosocial en jóvenes de esta edad. Entre los conflictos presentados por estos individuos existían problemas de funcionamiento académico y desórdenes de tipo psiquiátrico, incluyendo Asperger, trastornos de la personalidad y criminalidad $^{(8)}$. 


\section{" Nutrición Sensorial y Búsqueda de Sensaciones "}

Otros estudios efectuados con niños con problemas motores también apoyan el concepto que en la adolescencia, estos individuos se perciben a sí mismos como menos competentes, con menos apoyo social, con más alta ansiedad y menor estima personal que los grupos control de la misma edad, especialmente si el problema motor es severo ${ }^{(9)(10)(11)}$.

Otras investigaciones relacionadas con problemas de integración sensorial que se observan en la literatura se refieren a los buscadores de sensaciones peligrosas. Zuckerman describió esta situación como un estado definido por la necesidad de variedad, novedad y sensaciones complejas; y la voluntad para tomar riesgos físicos y sociales para obtener estas experiencias. Este investigador describe una tendencia a buscar situaciones estimulantes, que está basada en la individualidad biológica y genética, posteriormente influenciada por el ambiente $\stackrel{(12)}{ }$.

Se considera que los buscadores de sensaciones peligrosas intentan alcanzar un nivel óptimo de excitación, a través de actividades que pueden ser arriesgadas y que proporcionan una alta intensidad de input sensorial, algunas de las cuales pueden incluir escalar montañas, lanzarse en paracaídas, el vandalismo en el fútbol, el rafting e, incluso, el uso de drogas, alucinógenos y alcohol.

Los buscadores de sensaciones intensas son individuos que andan tras una variedad de experiencias novedosas, complejas e intensas, a través de actividades que ofrecen emoción y aventura. En muchos casos están deseosos de arriesgarse para lograr experiencias sensoriales. Cuando esta búsqueda de sensaciones peligrosas esta ligada a la búsqueda de input sensorial de tipo proprioceptivo y vestibular; podría darse en niños con déficit de integración sensorial y déficit atencional. De hecho, existe literatura que describe la alta incidencia del uso de drogas ilegales entre los individuos diagnosticados con déficit atencional $\stackrel{(13)}{ }$.

Los niños con déficit en modulación sensorial o con baja respuesta al input sensorial, pueden presentar una búsqueda desenfrenada de input sensorial. De pequeños, estos niños se calman a sí mismo a través de brincos, saltos y carreras. Al crecer, buscan sensaciones que los nutran a través de los sentidos. Muchas veces, la búsqueda es mediante actividades de juego, pues en ellas el niño es libre de expresarse a sí mismo. Estas actividades sirven como estrategias a corto plazo o como proveedoras de sensación de bienestar a largo plazo. Algunas de estas ocupaciones pueden ser cotidianas, como correr, escalar, nadar. Otras son planeadas cuidadosamente durante meses, como escalar una montaña. En algunos casos, la necesidad por una sensación e input sensorial intenso no toma en cuenta el nivel de maestría que tiene la persona y se toman riesgos que no son necesarios, como manejar motocicletas a alta velocidad ${ }^{(3)}$.

La búsqueda de input sensorial a través de ocupaciones también puede ser canalizada en forma constructiva. Fanchiang, Terapeuta Ocupacional, describe la metamorfosis Ocupacional como la conexión entre las necesidades biológicas por cierta estimulación sensorial y la elección posterior de ocupaciones. Ella describe el caso de un niño con déficit de integración sensorial que eligió ser masajista, para así proveerse a sí mismo del input que necesitaba para funcionar óptimamente. En este caso, el aporte de los padres en la estructura racional y elección de la ocupación se consideró muy importante. Así como los padres ayudan a estructurar y canalizar la elección de ocupaciones, también los terapeutas ocupacionales deben tomar en cuenta esta canalización de ocupaciones a largo plazo $\stackrel{(14)}{ }$. 


\section{" Organización de Tiempo y Espacio "}

Además de buscar sensaciones para mantener un estado de alerta, el individuo también debe organizar sus ocupaciones en el tiempo y el espacio. Individuos con déficit en praxis muchas veces presentan déficit en la organización del tiempo y el espacio; y estos problemas se hacen cada vez más evidentes a medida que la persona se desarrolla $\stackrel{(15)}{ }$.

Para Zemke, la organización de las ocupaciones en el tiempo y el espacio se agrega a las experiencias internas de la persona, para así reflejar el significado de la ocupación para la persona ${ }^{(16)}$. Por ejemplo, la persona que dedica muchas horas al trabajo en la oficina, refleja la importancia que ese trabajo tiene, o cuando una persona no es puntual podría reflejar la poca importancia que le da a esa cita. En el caso de las personas con déficit en praxis, el llegar tarde a una cita solo podría reflejar poca organización del tiempo y no debiera ser interpretada como una falta de interés en la cita.

Con relación al proceso de información sensorial, se puede ver que el tiempo es descrito como la percepción de cambio, incluyendo la percepción de simultaneidad, presente subjetivo, sucesión y duración ${ }^{(17)}$.

Esa noción de cambio y tiempo depende de la percepción de sucesión y duración $\stackrel{(18)}{\text {. }}$

Por lo tanto, es procesada a través de los sentidos. Los niños con problemas de integración sensorial tienen dificultad para procesar la información sensorial y, en consecuencia, también les resulta difícil procesar el paso del tiempo $\stackrel{(15)}{ }$.

De acuerdo a Zemke, el Sistema Nervioso Central participa en la estimación del tiempo a través de tres sistemas:

- El Sistema Troncoencefálico, que funciona en el presente y no separa el ser individual del ser de

otros.

El Sistema Límbico, que es la sensación emocional del tiempo. En este caso, tiempo y espacio son relacionados con otras personas.

- La Neocorteza, que anticipa, planea para el futuro y lleva a cabo el plan. ${ }^{(16)}$.

Cuando se revisa esta literatura es lógico deducir que niños que presentan dificultad en funciones biológicas y sensoriales o tienen disfunciones en el funcionamiento del SNC, van a manifestar dificultad en la percepción del tiempo y en la coordinación del tiempo con otros. Estos déficit pueden afectar puntualidad, organización de tareas en el trabajo, adjudicación de tiempo para cada tarea y la relación con otras personas cuando se debe organizar el tiempo.

La percepción del espacio a través de los sentidos ha sido altamente descrita en la literatura. Niños con déficit de integración sensorial pueden presentar déficit de percepción visual, vestibular y propioceptiva, que influyen sobre la forma como ellos perciben el espacio que los rodea.

La organización de ocupaciones en el tiempo y en el espacio se ven a lo largo de la vida. De pequeños se puede observar en la vestimenta o en la organización de los deberes escolares. En la juventud estos problemas se pueden observar en el uso de mapas, en la orientación espacial en grandes espacios como centros comerciales y edificios públicos, y en el orden de los espacios personales y compartidos.

De adultos, esta desorganización afecta la vida laboral y las relaciones sociales. En algunos casos tiende a depender de la sobre estructuración física o social y las personas aparecen como inflexibles. En otros casos, son seres creativos, que no son capaces de organizarse lo suficiente para que sus 
grandes obras sean reconocidas por el público. Como se puede apreciar, los déficits en percepción espacial y temporal afectan a largo plazo la organización de las ocupaciones a lo largo de la vida y la relación social con otros.

\section{" Nutrición Sensorial y Organización en el Tiempo en el Espacio: Aplicación a la Práctica Clínica.}

En esta sección se expondrán dos ejemplos:

Juan, puede ser catalogado como un buscador de sensaciones peligrosas. De niño no fue diagnosticado con déficit de integración sensorial, aunque en la actualidad él recuerda muchos signos que así lo indicaban. Relata que ataba una soga de los postes de la escalera y luego se colgaba de ella causando accidentes en algunas ocasiones. Durante su adolescencia formó parte de una pandilla y para él era fácil entrar en altercados físicos con amigos. Durante ese tiempo tuvo numerosos encuentros con la policía, hasta que a los 16 años, un amigo lo llevó a escalar. Juan relata que desde ese momento en adelante su vida cambió y el escalar montañas se convirtió en su deporte favorito, porque en cierta forma le proporcionó las sensaciones que él buscaba. Su búsqueda de aventuras riesgosas se fue transformando y en la actualidad es dueño de un gimnasio para escaladores. Parte de su trabajo es cambiar rutas, lo que significa una diaria dieta de input propioceptivo y vestibular. En este caso, Juan encontró sus propias ocupaciones que lo nutren sensorialmente dentro del contexto natural de su vida diaria. En muchos casos los terapeutas ocupacionales deben ayudar al paciente a encontrar esas ocupaciones. Para eso deben trabajar dentro de la sala de TO tanto como en el contexto natural en el cual se mueve la persona.

El siguiente caso ilustra otro punto: A los cuatro años, Ernesto fue diagnosticado con un déficit en praxis. Sus funciones motoras fueron tratadas por terapeutas ocupacionales con buenos resultados. A medida que Ernesto crecía, las dificultades motoras eran menos percibidas, pero su desorganización se convirtió en su mayor dificultad. Aunque era un buen alumno, llegaba tarde a clases, escribía sus tareas a altas horas de la noche y no podía organizarse para llenar el formulario para entrar a la universidad. Ernesto es un gran artista que necesita desarrollar una estructura a través de un horario de trabajo y estudio. Conflictos como éste se presentan usualmente en niños que han sido dados de alta después de que el problema motor ha sido resuelto.

El rol del terapeuta en el caso de Ernesto no concluye en la niñez ni con el tratamiento del problema motor dentro de la sesión de TO. En este caso, es importante ayudarlo a desarrollar una estructura en el contexto natural de su vida diaria a través de un horario de trabajo y estudio. Esto puede incluir el uso de agendas y relojes con alarmas que lo alerten cuando debe de terminar una tarea y empezar otra (cómo manejar al trabajo). La organización espacial también depende del uso de una estructura como lugares específicos para cada objeto y para cada tarea, y el aprendizaje en el uso de técnicas de navegación espacial (mapas e itinerarios).

Los ejemplos de Juan y Ernesto pueden incitar al terapeuta ocupacional a ayudar al individuo con déficit en integración sensorial a encontrar actividades que le permiten organizarse y nutrirse sensorialmente durante el resto de su vida; y considerar las necesidades sensoriales como conductas adaptativas a largo plazo. Estas últimas son conductas que ayudan a la persona a escoger un repertorio de actividades que proveen equilibrio y promueven bienestar a través de la experiencia sensorial. Estas conductas refuerzan a la persona para resistir y manejar el stress de la vida diaria y permanecer sano. (2) (모

En muchos casos, las actividades son escogidas para aumentar o regular la información sensorial. Por ejemplo, escoger escalar como actividad de recreación puede sugerir una búsqueda de input sensorial proprioceptivo, o escoger escuchar música clásica a través de audífonos, puede sugerir un intento a disminuir el input sensorial que se recibe diariamente. En su celebre libro "Homo Ludens", Hiuzinga planteó que lo lúdico es algo muy serio y que trasciende el campo de la mera diversión. El dice que el 
ser humano que no juega no desarrolla una dimensión fundamental de su existencia. A través del juego, las personas son capaces cambiar el estado anímico y en este proceso las actividades se convierten en una forma de terapia para superar una serie de dificultades $\underline{(20)}$.

\section{" Conclusión "}

El estilo de procesar información persiste a lo largo de la vida así también persisten los déficit de integración sensorial. Tanto el estilo de procesar información como los déficit de integración sensorial influyen la elección de ocupaciones de cada persona. La necesidad de estímulos sensoriales se vislumbra mas claramente en ocupaciones de elección libre como son las actividades de juego y recreación. Generalmente, a través de las ocupaciones de juego, recreación y proceso ${ }^{(3)}{ }^{(19)}$ los seres humanos nos centramos más en el proceso que en el producto. Se trata de disfrutarlo sin pensar en los resultados, por lo tanto en estas ocupaciones de proceso la persona se nutre sensorialmente.

Es por eso que las ocupaciones de juego y recreación se pueden utilizar terapéuticamente en personas que durante su infancia tuvieron un diagnóstico de déficit de integración sensorial, ya sea como dispraxias o como dificultades de modulación. Habitualmente, se trata de experiencias que el individuo había desarrollado durante su infancia y que vuelven a conectarlo consigo mismo.

En consecuencia, es fundamental que los padres, profesores y terapeutas que se relacionan con el niño que presenta problemas en déficit integración sensorial, estén bien informados y se preocupen de que el pequeño sea correctamente evaluado y tratado en todos los aspectos de la vida diaria. Parte de la evaluación y tratamiento es encontrar esa actividad de juego y recreación que lo nutriría sensorialmente y lo apoye en el crecimiento. Eso significa que desde el principio en el proceso de intervención, tratemos al niño en el contexto natural de la vida diaria tanto como dentro del contexto de la sala de tratamiento. Al no recibir apoyo y ayuda terapéutica en ambos contextos, la persona enfrentará dificultades cada vez mayores y perderá confianza en sí misma, limitándose innecesariamente en sus actividades como adulto.

\section{- Referencias =}

- (1) Clark, F., Wood, W., \& Larson, E. (1998). Occupational science: Occupational therapy's legacy for the 21th century. Willard and Spackcman's Occupational Therapy (pp.13 - 21). Philadelphia, PA: J.B. Lippincott Company.

Yerxa, E. (1998). Health and the human spirit for occupation. The American Journal of Occupational Therapy, 52 (6) 412 - 418.

Blanche, E. (2002). Play and process: Adult play embedded in the daily routine. In J. Roopnarire (Ed.). Conceptual, social-cognitive, and contextual issues in the field of play. Vol. 4 Westport, Conn: Ablex Publishing.

Ermer, J. and Dunn, W. (1998). The sensory profile: A discriminant analysis of children with and without disabilities. The American Journal of Occupational Therapy, 52 (4), 283 - 290.

Miller, L., Reisman, J. , McIntosh, D., \& Simon, J. (2001). An ecological model of sensory modulation performance of children with fragileX syndrome, autism, attention deficit with hyperactivity and sensory modulation disorder. In Smith-Roley, S. Blanche, E. \& Schaaf, R. (Eds) (2001). Sensory integration with diverse populations. San Antonio, 
Psychological Corporation.

(6)

Mulligan, S. (1996). An analysis of score patterns of children with attention disorders on the sensory integration and praxis tests. The American Journal of Occupational Therapy. 50 (8), 647 - 654.

(7)

Parham, L. D. (1998). The relationship of sensory integrative development to achievement in elementary students: Four-year longitudinal patterns. Occupational Therapy Journal of Research, 18(3), 105-127.

Rasmussen, P. \& Gillberg, C. (2000). Natural outcome of ADHD with developmental coordination disorder at age 22 years: a controlled, longitudinal, community-based study. J Am Acad Child Adolesc Psychiatry. 39(11):1424-3.

Cantell, M.H., Smyth, M.M., Ahonen, T.P. (2003). Two distinct pathways for developmental coordination disorder: persistence and resolution. Hum Mov Sci. 22 (4-5):413-3.

Skinner, R.A. \& Piek, J.P. (2001) Psychosocial implications of poor motor coordination in children and adolescents. Hum Mov Sci. 20 (12):73-94.

Dewey D, Kaplan BJ, Crawford SG, Wilson BN. (2002). Developmental coordination disorder: associated problems in attention, learning, and psychosocial adjustment. Hum Mov Sci. 21(5-6):905-18.

Zuckerman, M. (1994). Behavioral expressions and biosocial bases of sensation seeking. Cambridge: University Press.

Horner BR, Scheibe KE. (1997) Prevalence and implications of attention-deficit hyperactivity disorder among adolescents in treatment for substance abuse. J Am Acad Child Adolesc Psychiatry. 36(1):30-6.

Fanchiang, S. (1996). The other side of the coin: Growing up with a learning disability. American Journal of Occupational Therapy, 50, 277285.

Blanche, E.I. and Parham, D. (2001). Praxis and the organization of behavior in time and space. In Smith-Roley, S. Blanche, E. \& Schaaf, R. (Eds) (2001). Sensory integration with diverse populations. San Antonio, Psychological Corporation.

Zemke, R. (2004). Time, Space and the Kaleidoscopes of Occupation. Eleanor Clarke Slagle Lecture, 2004 AOTA Conference, Minneapolis, MN.

Poppel, E. (1988). Time perception. In J.M. Wolfe (Ed.), Readings from the Encyclopedia of Neuroscience: Sensory systems II-Senses other than vision (pp. 134-135). Boston: Birkhauser.

Fraisse, P. (1963). The psychology of time. New York: Harper and Row.

Imperatore, E. I. (2002). La estamos pasando bien? El juego del adulto. 
Santiago, Chile: Editorial Grijalbo.

(20) Huizinga, J. (1950). Homo ludens: A study of the play element in culture. Boston: Beacon Press. (Original work published in 1938). 